\title{
Effects of a New Combination of Cranberry Extracts, D-Mannose and GAGs for the Management of Uncomplicated Urinary Tract Infection
}

Stefano Manno ${ }^{1 *}$, Cicione Antonio ${ }^{2}$, Dell'Atti Lucio ${ }^{3}$, Capretti Camilla ${ }^{3}$, Scarcella Simone ${ }^{3}$, Cantiello Francesco ${ }^{1}$ and Damiano Rocco $^{1}$

${ }^{1}$ Urology Unit, Magna Graecia University of Catanzaro, Catanzaro, Italy

${ }^{2}$ Urology Unit, Ospedale Città di Castello, ASL Umbria 1, Città di Castello, Italy

${ }^{3}$ Department of Urology, Polytechnic University of Marche Region, University Hospital “Ospedali Riuniti”, Ancona, Italy

*Corresponding Author: Stefano Manno, Magna Graecia University, Viale Europa, 88100, Catanzaro, Italy; Email: stefanomanno85@gmail.com

Received: January 08, 2019; Accepted: January 19, 2019; Published: February 09, 2019;

\begin{abstract}
OBJECTIVE: Several studies have investigated the role of cranberry extract and D-mannose in the prevention of recurrent urinary tract infections (UTIs). The aim of this preliminary study was to test whether the use of a new dietary supplement (UROIAL ${ }^{\mathrm{TM}}$ sachets) containing cranberry extracts (S\&R PACs), D-mannose, hyaluronic Acid and Glucosamine Chondroitin may reduce the incidence of episodes of cystitis and improve urinary symptoms.
\end{abstract}

METHODS: In this prospective comparative study, 40 women with an acute diagnosis of cystitis received a single sachet of Fosfomycin Tromethamine (3gr). The subjects were then randomly assigned to two groups: Group A: 20 women were given UROIAL ${ }^{\mathrm{TM}}, 2$ sachet per day during the first 7 days, then 1 sachet per day for two weeks; Group B: 20 women did not receive any treatment to serve as a control group.

RESULTS: The results of the present study showed a complete remission of urinary symptoms in 37 women, a slight decrease in urinary symptoms was observed in 2 subjects, whereas 1 woman who stopped the treatment was considered a drop-out. Patients in Group A had a lower incidence of episodes of recurrent cystitis during treatment and follow-up; urine samples had significantly lower median bacterial load compared to baseline as well as a symptomatic relief was reported in treated subjects despite the control group.

DISCUSSION: Several studies have investigated and demonstrated the role of cranberry extracts in the prevention of recurrent urinary tract infections (UTIs), on different selected subpopulations and even at increased risk of UTI. Even the use of D-mannose, a natural sugar has proven to have clinical benefit, although clinical data is limited. A randomized placebo-controlled non-blinded clinical trial has shown that a daily dose of $2 \mathrm{~g}$ d-mannose was superior to placebo in preventing UTI. Mannose receptors are found on uroepithelial cells lining the urinary tract they constitute the protective mucopolysaccharide layer of the bladder. The mechanism of action involves binding of the mannose receptors to E. coli pili, thus preventing both adhesion to and invasion of urothelial cells. The interaction between mannose molecules and E. coli bacteria can then be washed away with urine voiding.

CONCLUSIONS: Our data indicate that the addition of GAGs (hyaluronic acid and chondroitin sulfate) to cranberry/D-Mannose containing products could represent a valid novel therapeutic approach for the treatment and/or prophylaxis of cystitis.

Keywords: Uncomplicated UTI, Cranberry, D-Mannose and GAGs

\section{Introduction}

Urinary Tract Infections (UTIs) are among the most common infectious diseases and almost exclusively caused by bacteria (1). UTIs refers to the presence of a certain number of bacteria in the urine (generally $>10^{5} / \mathrm{ml}$ ) and symptomatic UTIs are classified in order of severity as upper UTI, with infection in the kidney and lower UTI (cystitis) with bacteria into the bladder $(1,2)$. Clinically, UTIs classification comprises either uncomplicated or complicated cases, depending on the presence of structural or neurological urinary tract abnormalities (3). According to the latest data, UTIs affect nearly half of all women, and it is estimated that around $11 \%$ of women aged over 18 have an UTI each year (4-6).
Based on severity can also be classified as uncomplicated or complicated. This distinction is useful to guide the choice and duration of antimicrobial treatment. In particular, uncomplicated UTIs occur in patients with a structurally and functionally normal urinary tract. In contrast, complicated UTIs are characterized by functional or anatomical abnormalities within the urinary tract (7).

Recurrent UTIs occur more commonly in healthy women who have anatomically and functionally normal urinary tracts (8).

In uncomplicated cases, recurrent UTIs are treatable with a shortcourse of antibiotics, but the inappropriate use of these antimicrobial agents has been accompanied by the rapid spread of resistant bacterial 
strains (9). The prevalent pathogenic bacteria that cause UTIs are Gram-negative pathogens and the most frequently isolated bacterial strain is Escherichia coli, representing $>80 \%$ of infections $(10,11)$.

Bacterial cystitis (also called acute cystitis) can occur in both women and men and some people develop recurrent infections of the urinary tract (12). Three or more urinary tract infections within 12 months define the recurring UTI, as well as two or more recurrences within 6 months. The same bacterial species that caused previous infection is typically responsible for relapses. Approximately $20-30 \%$ of adult women with an initial UTI will experience a recurrence within 3-4 months.

The aim of this preliminary study was to verifying whether the use of a new dietary supplement (UROIAL ${ }^{\mathrm{TM}}$ sachets) containing type-A proanthocyanidins (S\&R PACs), D-mannose, hyaluronic Acid and Glucosamine Chondroitin can be of use in reducing the incidence of episodes of recurrent cystitis and the elimination of urinary symptoms.

\section{Material and Methods}

\section{Study Design}

A randomized parallel group intervention trial was designed to evaluate the prophylactic effects of a novel formulation of cranberry extracts combined with d-mannose and hydronic acid on female subjects with a history of recurrent UTIs presenting with uncomplicated cystitis. Of 42 eligible women, two were excluded because they did not meet the inclusion criteria (Fig. 1). Thus, 40 women were randomly allocated and 20 treated with Cranberry, D-Mannose, Vitamin C and Hyaluronic acid; Group A. The duration of the intervention was 12 weeks and the end of follow-up was 3 months after the start of treatment. All participants attended regular clinical appointments.

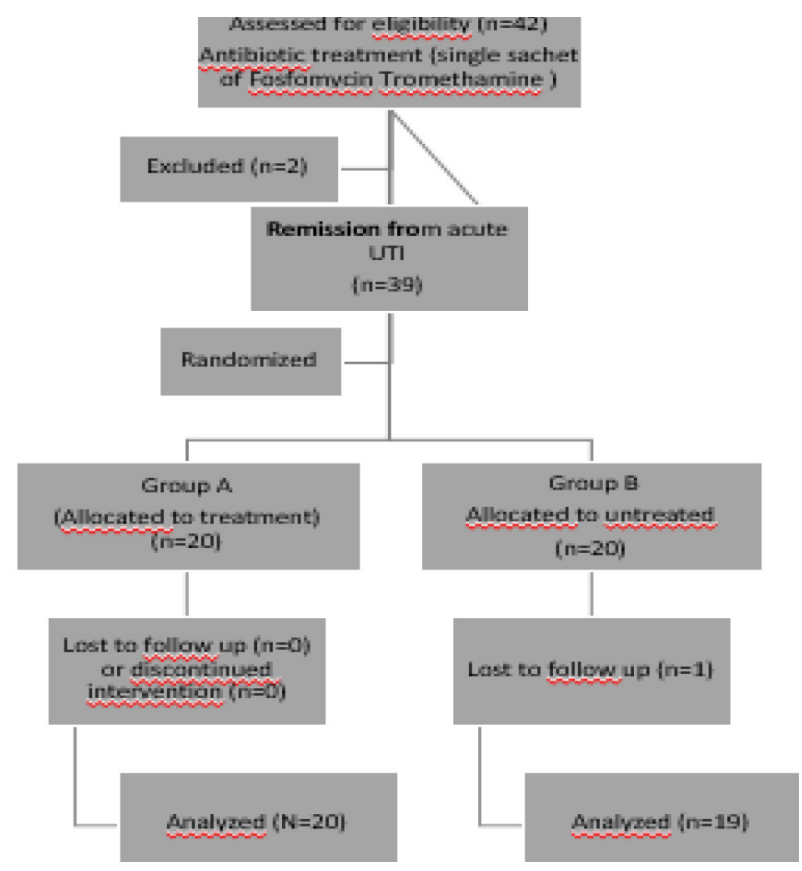

Figure 1. Flow diagram of enrolment, intervention allocation and data analysis.

\section{Subjects}

Eligible subjects were all adult Caucasian females affected by acute uncomplicated cystitis and all had a history of recurrent UTIs. The participants were enrolled (recruitment and treatment) between August 2017 and December 2017 and after antibiotic treatment with Fosfomycin 1 sachet $\left(\mathrm{UROFOS}^{\circledast}\right)$, were randomly assigned to the two different groups. The inclusion criteria were as follows: (1) two or more episodes of UTI in the last 12 months documented by urine culture; and (2) at least one urinary symptom and/or positive urinary nitrate test or leukocyturia. Women were ineligible if any of the following criteria were present:

(1) pregnancy or lactation; (2) abnormalities of the upper urinary tract, including the presence of urinary stones; (3) a permanent urinary catheter; (4) complete urinary incontinence; (6) stage 5 chronic kidney disease (glomerular filtration rate $(\mathrm{GFR})<15 \mathrm{ml} / \mathrm{min}$ ). The study took place at the University of Magna Graecia (CZ), Italy.

\section{Nutraceutical Administration}

The tested compound is a commercially available as a dietary supplement marketed in Italy, UROIAL (S\&R Farmaceutici S.p.A, Bastia Umbra, Perugia, Italy) containing the following ingredients per dose respectively: S\&R PACs (250mg) with type-A proanthocyanidins (72 mg), d-mannose (1000 mg), chondroitin sulfate (200mg), vitamin $\mathrm{C}(120 \mathrm{mg})$ and hyaluronic acid $(100 \mathrm{mg})$. Patients were advised to take 2 sachets for 2 weeks and one sachet for another two weeks.

\section{Laboratory Assessments}

Urinalyses and cultures were performed using standard methods, as previously described (13). Diagnosis of UTIs was based on 105 colony-forming units (CFU) in $1 \mathrm{ml}$ of urine sample. Urinary specimens were streaked for isolation on MacConkey agar and blood agar plates for bacterial separation. Positive urine culture results, Gram staining and morphology of pre-dominant colonies were defined according to the guidelines of the Clinical and Laboratory Standards Institute (CLSI).

\section{Statistical analysis}

Clinical data (bacterial count) were analysed by the nonparametric One-Way ABnova Analysis. p value $<0.05$ was considered statistically significant.

\section{Results}

The study included a total of 40 women with acute cystitis and a history of recurrent cystitis episodes. All but one patient had a complete remission of urinary symptoms after antibiotic treatment with fosfomycin. A slight decrease in urinary symptoms was observed in 2 subjects, whereas 1 woman who stopped the treatment was considered a drop-out. In fact, 38 women participants completed the 12-week intervention period (flow diagram). Table 1 shows the number of symptomatic episodes and number of recurrences recorded during the observation period starting from week 4. Patients in Group A had a lower incidence of episodes of recurrent cystitis during treatment and follow-up and urine samples had a significantly 
lower median bacterial load compared to baseline. Group A showed a greater number of negative urine cultures compared to group B. Indeed, a higher microbial count (105 and/or $>105 \mathrm{CFU} / \mathrm{ml})$ was more frequently observed in the urine cultures of group B (Table 2). Complete symptomatic relief was reported in the majority $(85 \%)$ of patients in Group A while only $10 \%$ was reported by subjects in the untreated control group.

Table 1. Number patients with symptomatic episodes and recurrences of cystitis during follow-up (12 weeks)

\begin{tabular}{|l|c|c|c|c|}
\hline & \multicolumn{2}{|c|}{ Symptomatic episodes } & \multicolumn{2}{c|}{ Recurrences } \\
\hline & 4 weeks & 12 weeks & 4 weeks & 12 weeks \\
\hline Group A & $1(20)$ & $3(20)$ & $0(20)$ & $0(20)$ \\
\hline Group B & $5(19)$ & $17(19)$ & $2(19)$ & $3(19)$ \\
\hline
\end{tabular}

Table 2. Positive E.coil bacterial Counts during follow-up 12 weeks.

\begin{tabular}{|l|c|c|}
\hline & Baseline & 12 weeks \\
\hline Group A & $15(20)$ & $1(20)^{*}$ \\
\hline Group B & $16(19)$ & $10(19)$ \\
\hline
\end{tabular}

\section{Discussion}

Several studies have investigated and demonstrated the role of cranberry extracts in the prevention of recurrent Urinary Tract Infections (UTIs), on different selected subpopulations and even at increased risk of UTI (14). Cranberries contain a high content of type A pro-antho-cyanidins whose urinary metabolites interfere with the adhesiveness of uro-pathogenic bacteria to the bladder epithelium, thus preventing adherence of bacteria to the bladder lining $(15,16)$. They are not as effective as antibiotics and have variable intestinal absorption (17). One RCT showed that both cranberry tablets and juice were able to significantly reduce the proportion of women experiencing rUTI over the 12 months compared with placebo (18\% vs. $32 \%$ ) (18). A Cochrane database systematic review updated in 2012 looked at 4,473 participants (19). It showed that there was no significant reduction in the occurrence of symptomatic UTI overall or for any subgroups tested (20). These included children, the elderly, women, pregnant women, cancer patients, neuropathic bladder patients or spinal patients. The review concluded that with additional studies included the evidence for cranberry juice in preventing UTIs was less than previously thought. There were, however, three studies that showed cranberry products were equivalent to antibiotics in preventing UTIs, with less side effects. These were limited by the lack of standardization between tablets and powders and the limitations associated with lack of information about the active ingredient present in each sample. No recommendations were able to be made.

Even the use of D-mannose, a natural sugar has proven to have clinical benefit, although clinical data is limited. A randomized placebo-controlled non-blinded clinical trial has shown that a daily dose of $2 \mathrm{~g}$ d-mannose was superior to placebo in preventing UTI (21). Mannose receptors are found on uroepithelial cells lining the urinary tract they constitute the protective mucopolysaccharide layer of the bladder. The mechanism of action involves binding of the mannose receptors to E. coli pili, thus preventing both adhesion to and invasion of urothelial cells. The interaction between mannose molecules and $\mathrm{E}$. coli bacteria can then be washed away with urine voiding $(2,21,22)$.

Vitamin $\mathrm{C}$ can acidify urine and has been weakly associated with decreased risk of UTI (23). There is data to suggest that vitamin C can also have a bacteriostatic effect and be effective in treatment and prevention of UTI in pregnant women (24).

Moreover, recently several non-antimicrobial options for rUTIs have been introduced, such as oral or Intra-vesical instillation of glycosaminoglycans (GAGs), which are able to strengthen bladder defense mechanisms. In particular intravesical administration of these GAGs, in particular hyaluronic acid (HA) and chondroitin sulfate (CS), have proven to be successful for both interstitial and recurrent bacterial cystitis (25-27). Glycosaminoglycan (GAG) layer replenishment con be exploited for many indications, including interstitial cystitis, overactive bladder syndrome, radiation cystitis and prevention of rUTI. At present, intra-vesical therapies are reserved for only those with the most unresponsive rUTIs (26). Previous studies by our research group looked at 57 women with rUTI and randomized them to either $50 \mathrm{~mL}$ of combination $1.6 \%$ hyaluronic acid and $2 \%$ chondroitin or treatment with $50 \mathrm{~mL}$ placebo. The treatment arm significantly reduced UTI rates over the 12-week follow-up $(-86.6 \%$ vs. $-9.6 \%)(28)$. Thus, a management plan including a combination of a non-antimicrobial and selective antimicrobial regime should be considered, such as a novel formulation under investigation in the present study.

\section{Conclusion}

Management of UTI should be individualized and symptombased, but tends to follow a progressive therapeutic ladder. Clinicians should remember to include alternative therapeutics for support and management that should be aimed at improving quality of life (29).

This pilot study reveals that the nutraceutical formulation containing type A PACs from Cranberry extracts, D-Mannose, Vitamin C, Hyaluronic and Chondroitin sulfate (UROIAL ${ }^{\circledR}$ ) is a valid therapeutic approach in reducing the incidence of episodes of recurrent cystitis and in the elimination of urinary symptoms. Our data indicate that the addition of GAGs to cranberry/D-Mannose containing products could represent a valid novel therapeutic approach for the treatment and/or prophylaxis of cystitis.

\section{References}

1. Foxman B. Urinary tract infection syndromes: occurrence, recurrence, bacteriology, risk factors, and disease burden. Infect Dis Clin North Am. 2014; 28: 1-13.

2. Smelov V, Naber K, Bjerklund Johansen TE. Diagnostic Criteria in Urological Diseases do not Always Match with Findings by Extended Culture Techniques and Metagenomic Sequencing of 16S rDNA. Open Microbiol J. 2016 Feb 29; 10: 23-6. doi: 10.2174/1874285801610010023. eCollection 2016.

3. Zacchè MM, Giarenis I.Therapies in early development for the treatment of urinary tract inflammation. Expert Opin Investig Drugs. 2016; 25(5): 531-40. Epub 2016 Mar 24. Review.

4. Foxman B, Brown P. Epidemiology of urinary tract infections: transmission and risk factors, incidence, and costs. Infect Dis Clin North Am. 2003; 17: 227-41.3

5. Salvatore S, Salvatore S, Cattoni E, Siesto G, Serati M, Sorice P, et al. Urinary tract infections in women. Eur J Obstet Gynecol Reprod Biol. 2011; 156: 131-6.

6. Tandogdu Z, Wagenlehner FM. Global epidemiology of urinary tract infections. Curr Opin Infect Dis. 2016; 29: 73-9 
7. Hooton TM. Clinical practice. Uncomplicated urinary tract infection. N Engl J Med. 2012; 366: 1028-1037.

8. Glover M, Moreira CG, Sperandio V, Zimmern P. Recurrent urinary tract infections in healthy and nonpregnant women. Urol Sci. 2014; 25 : 1-8.

9. Pallett A, Hand K. Complicated urinary tract infections: practical solutions for the treatment of multiresistant.

10. Hooton TM, Stamm WE. Diagnosis and treatment of uncomplicated urinary tract infection. Infect Dis Clin North Am. 1997; 11: 551-81.

11. Stamm WE, Hooton TM. Management of urinary tract infections in adults. N Engl J Med. 1993; 329: 1328-34.

12. Fiore DC, Fox CL. FP. Urology and nephrology update: recurrent urinary tract infection. Essent. 2014 Jan; 416: 30-7.

13. Clinical and Laboratory Standards Institute (CLSI; formerly NCCLS). Urinalysis and Collection, Transportation, and Preservation of Urine Specimens; Approved Guideline. 2nd ed. Vol. 21. No. 19. Document Eur Urol. 2011 Apr; 59(4): 645-51. doi: 10.1016/j.eururo.2010.12.039. Epub 2011 Jan 18. Erratum in: Eur Urol. 2011 Jul; 60(1): 193. GP-16A2. Wayne (PA); 2001.

14. Wing DA, Rumney PJ, Preslicka CW, Chung JH Daily cranberry juice for the prevention of asymptomatic bacteriuria in pregnancy: a randomized, controlled pilot study. J Urol. 2008 Oct; 180(4): 1367-72

15. Sanchez Ballester F, Ruiz Vidal V, Lo' pez Alcina E, et al. M. Cysticleans a highly pac standardized content in the prevention of recurrent urinary tract infections: an observational, prospective cohort study. BMC Urol. 2013; 13: 28.

16. Gupta K, Chou MY, Howell A, et al. Cranberry products inhibit adherence of p-fimbriated Escherichia coli to primary cultured bladder and vaginal epithelial cells. J Urol. 2007; 177: 2357-2360

17. Beerepoot MA, ter Riet G, Nys S, van der Wal WM, de Borgie CA, de Reijke TM, Prins JM, Koeijers J, Verbon A, Stobberingh E, Geerlings SE. Cranberries vs antibiotics to prevent urinary tract infections: a randomized double-blind noninferiority trial in premenopausal women. Arch Intern Med. $2011 \mathrm{Jul} 25$; 171(14): 1270-8.

18. Stothers L. A randomized trial to evaluate effectiveness and cost effectiveness of naturopathic cranberry products as prophylaxis against urinary tract infection in women.Can J Urol. 2002 Jun; 9(3): 1558-62.

19. Jepson RG, Williams G, Craig JC. Cranberries for preventing urinary tract infections. Cochrane Database Syst Rev. 2012 Oct 17; 10: CD001321
20. Kranjčec B, Papeš D, Altarac S. D-mannose powder for prophylaxis of recurrent urinary tract infections in women: a randomized clinical trial. World J Urol. 2014 Feb; 32(1): 79-84.

21. Wellens A, Garofalo C, Nguyen H, Van Gerven N, Slättegård R, Hernalsteens JP, Wyns L, Oscarson S, De Greve H, Hultgren S, Bouckaert J. Intervening with urinary tract infections using anti-adhesives based on the crystal structure of the FimH-oligomannose-3 complex. PLoS One. 2008 Apr 30; 3(4): e2040. doi: 10.1371/journal.pone.0002040. Erratum in: PLoS ONE. 2008; 3(6).

22. Martinez JJ, Hultgren SJ. Requirement of Rho-family GTPases in the invasion of Type 1-piliated uropathogenic Escherichia coli. Cell Microbiol. 2002 Jan; 4(1): 19-28.

23. Foxman B, Chi JW. Health behavior and urinary tract infection in college-aged women. J Clin Epidemiol. 1990; 43(4): 329-37.

24. Ochoa-Brust GJ, Fernández AR, Villanueva-Ruiz GJ, Velasco R, TrujilloHernández B, Vásquez C Daily intake of $100 \mathrm{mg}$ ascorbic acid as urinary tract infection prophylactic agent during pregnancy. Acta Obstet Gynecol Scand. 2007; 86(7): 783-7.

25. Nickel JC, Hanno P, Kumar K, Thomas H. Second multicenter, randomized, double-blind, parallel-group evaluation of effectiveness and safety of intravesical sodium chondroitin sulfate compared with inactive vehicle control in subjects with interstitial cystitis/bladder pain syndrome. Urology 2012; 79(6): 1220-12244.

26. Damiano R, Cicione A. The role of sodium hyaluronate and sodium chondroitin sulphate in the management of bladder disease. Ther Adv Urol. 2011 Oct; 3(5): 223-32.

27. Cicione A, Cantiello F, Ucciero G, Salonia A, Madeo I, Bava I, Aliberti A, Damiano R. Int J Urol. 2014 Aug; 21(8): 763-8. doi: 10.1111/iju.12430. Epub 2014 Mar 19 Restoring the glycosaminoglycans layer in recurrent cystitis: experimental and clinical foundations.

28. Damiano R, Quarto G, Bava I, Ucciero G, De Domenico R, Palumbo MI, Autorino R.

29. Prevention of recurrent urinary tract infections by intravesical administration of hyaluronic acid and chondroitin sulphate: a placebo-controlled randomised trial.

30. Douglas-Moore JL, Goddard J. Current best practice in the management of cystitis and pelvic pain. Ther Adv Urol. 2017 Oct 25; 10(1): 17-22.

\section{Citation:}

Stefano Manno, Cicione Antonio, Dell'Atti Lucio, Capretti Camilla, Scarcella Simone, Cantiello Francesco and Damiano Rocco (2019) Effects of a New Combination of Cranberry Extracts, D-Mannose and GAGs for the Management of Uncomplicated Urinary Tract Infection. Endocrinol Diabetes Metab $J$ Volume 3(1): 1-4. 